\title{
Novel approach to binary dynamics: application to the fifth post-Newtonian level
}

\author{
Donato Bini ${ }^{1,2}$, Thibault Damour ${ }^{3}$, Andrea Geralico ${ }^{1}$ \\ ${ }^{1}$ Istituto per le Applicazioni del Calcolo "M. Picone," CNR, I-00185 Rome, Italy \\ ${ }^{2}$ INFN, Sezione di Roma Tre, I-00146 Rome, Italy \\ ${ }^{3}$ Institut des Hautes Études Scientifiques, 91440 Bures-sur-Yvette, France.
}

(Dated: September 6, 2019)

\begin{abstract}
We introduce a new methodology for deriving the conservative dynamics of gravitationally interacting binary systems. Our approach combines, in a novel way, several theoretical formalisms: post-Newtonian, post-Minkowskian, multipolar-post-Minkowskian, gravitational self-force, and effective one-body. We apply our method to the derivation of the fifth post-Newtonian dynamics. By restricting our results to the third post-Minkowskian level, we give the first independent confirmation of the recent result of Bern et al. [Phys. Rev. Lett. 122, 201603 (2019)]. We also offer checks for future fourth post-Minkowskian calculations. Our technique can, in principle, be extended to higher orders of perturbation theory.
\end{abstract}

Introduction.-The detection of the coalescence of compact binaries by the LIGO-Virgo collaboration [1] demands an ever more accurate knowledge of the gravitational dynamics and radiation of binary systems. We propose here a new methodology for improving the theoretical description of the conservative dynamics of twobody systems in General Relativity. This methodology unifies in a novel way an array of previously developed theoretical tools, and combines it with some new insights. This allows one to reach in an expedient manner new high-order results of direct physical significance. Here, we exemplify the efficiency of our method by applying it to the first (essentially complete) computation of the conservative two-body dynamics (of two non-spinning masses $\left.m_{1}, m_{2}\right)$ at the fifth post-Newtonian (5PN) accuracy, i.e. one order in $(v / c)^{2}$ beyond the last postNewtonian (PN) order at which this dynamics has been heretofore fully derived [2-8]. [Our 5PN-level result cannot be compared with the recent $5 \mathrm{PN}$-level works $[9,10]$, because the latter have computed only the small, and non gauge-invariant, subset of "static" contributions to the 5PN Hamiltonian.] As a by product of our calculation, we also compute the $5 \mathrm{PN}$-level contribution to the (gauge-invariant) scattering angle of two bodies considered at the third post-Minkowskian (3PM) approximation. We find a result which is in agreement with the corresponding result recently derived from a two-loop scattering amplitude computation [11, 12], thereby providing the first confirmation of the latter result going beyond the $4 \mathrm{PN}-$ level checks derived from the gauge-invariant $4 \mathrm{PN}$ scattering [13]. The result presented here is only a first application of a general methodology which can be extended to higher PN orders.

Let us motivate our new approach by considering the state of the art of the general relativistic two-body problem. The PN formalism has been the method of choice, during many years, for analytically tackling the dynamics of binary systems. However, it has recently reached a level of complexity which renders further progress acutely difficult. Most of the technical efficiency of the PN formalism comes from the fact that it systematically re- places the four-dimensional relativistic propagator

$$
P_{4}\left(t, \mathbf{x}, t^{\prime}, \mathbf{x}^{\prime}\right) \equiv \square^{-1}=\left(\Delta-c^{-2} \partial_{t}^{2}\right)^{-1},
$$

entering the post-Minkowskian (PM) formalism, by its formal expansion in inverse powers of the velocity of light:

$$
P_{4}^{\mathrm{PN}}\left(t, \mathbf{x}, t^{\prime}, \mathbf{x}^{\prime}\right)=\left(\Delta^{-1}+\frac{1}{c^{2}} \partial_{t}^{2} \Delta^{-2}+\cdots\right) \delta\left(t-t^{\prime}\right) .
$$

Here, we consider the time-symmetric propagator (as appropriate to the derivation of the conservative dynamics). Each term on the right-hand side (RHS) of the PN expansion (2) of $P_{4}\left(t, \mathbf{x}, t^{\prime}, \mathbf{x}^{\prime}\right)$ is local-in-time, in the sense that it involves a derivative of $\delta\left(t-t^{\prime}\right)$. Inserting the PN expansion (2) in the reduced action describing (after having integrated out the gravitational field) the PM-expanded dynamics of two worldlines [14] allows one to express the relativistic gravitational interaction of two particles in terms of iterated integrals (given by Feynman-like diagrams) involving only the concatenation of the instantaneous three-dimensional propagator

$$
P_{3}\left(t, \mathbf{x}, t^{\prime}, \mathbf{x}^{\prime}\right)=\delta\left(t-t^{\prime}\right) \Delta^{-1}=-\frac{1}{4 \pi} \frac{\delta\left(t-t^{\prime}\right)}{\left|\mathbf{x}-\mathbf{x}^{\prime}\right|},
$$

or of its descendants $\frac{1}{c^{2}} \partial_{t}^{2} \Delta^{-2} \delta\left(t-t^{\prime}\right)+\cdots$. The use of such a PN-expanded propagator, together with the corresponding PN simplification of the nonlinear vertices generated by the Einstein-Hilbert action, leads to drastic simplifications (compared to a corresponding PM-expanded action involving the original 4-dimensional propagator $\left.P_{4}^{\mathrm{PN}}\left(t, \mathbf{x}, t^{\prime}, \mathbf{x}^{\prime}\right)\right)$ in the computation of the reduced action, especially when using the Arnowitt-DeserMisner Hamiltonian approach [15]. Indeed, the concatenated massless propagators $\Delta^{-n}$ lead to 3-dimensional integrals containing only one length scale, namely (in $\mathbf{x}$-space) the distance $r_{12} \equiv\left|\mathbf{x}_{1}-\mathbf{x}_{2}\right|$ between the two bodies.

However, as had been anticipated years ago 16], the PN approach undergoes a fundamental conceptual (and technical) breakdown at the fourth post-Newtonian $(4 \mathrm{PN})$ level. At this level the naive $\mathrm{PN}$ expansion 
(2) of the 4-dimensional propagator $P_{4}^{\mathrm{PN}}\left(t, \mathbf{x}, t^{\prime}, \mathbf{x}^{\prime}\right)$ becomes fundamentally inadequate because the gravitational interaction necessarily involves nonlocal-in-time effects that cannot be described in terms of the sequence of quasi-instantaneous terms appearing on the RHS of (2). This conceptual failure of the PN expansion leads, at the technical level, to the appearance of infrared logarithmic divergences in the formal computation of the PN-expanded action. The current direct perturbative computations (using the expansion (2)) of the 4PN-level reduced action [2 8] have succeeded in solving this problem in various ways. However, this variety of approaches, which included discrepant intermediate results [4] before complete agreement was reached, show that straightforward perturbative PN computations have reached their limit of easily verifiable reliability. This makes it urgent to develop a new methodology, as we do here. Our new approach consists of several steps that we explain in turn.

First step: computing the nonlocal-in-time piece of the action - The first step is to use results derived within the (PN-matched [16-18]) multipolar-post-Minkowskian (MPM) formalism [19] to decompose, at some given $\mathrm{PN}$ accuracy, the complete, (reduced) two-body conservative action $S_{\text {tot }}$ in two separate pieces: a nonlocal-in-time part, say $S^{\text {nonloc }}$, and a local-in-time part, $S^{\text {loc }}$ :

$$
\begin{aligned}
S_{\text {tot }}^{\leq n \mathrm{PN}}\left[x_{1}\left(s_{1}\right), x_{2}\left(s_{2}\right)\right] & =S_{\mathrm{loc}}^{\leq n \mathrm{PN}}\left[x_{1}\left(s_{1}\right), x_{2}\left(s_{2}\right)\right] \\
& +S_{\text {nonloc }}^{\leq n \mathrm{PN}}\left[x_{1}\left(s_{1}\right), x_{2}\left(s_{2}\right)\right] .
\end{aligned}
$$

Here, $S_{\text {tot }}\left[x_{1}\left(s_{1}\right), x_{2}\left(s_{2}\right)\right]$ is a time-symmetric functional of the two worldlines defined (before considering it approximate estimation at the $n \mathrm{PN}$ accuracy) by its PMexpansion [14]. The decomposition (44) makes sense, at a given PN accuracy, because the MPM formalism yields an efficient tool for computing the non-local piece $S_{\text {nonloc }}^{\leq n \mathrm{PN}}$. From Ref. [16] one knows that $S_{\text {nonloc }}^{\leq n \mathrm{PN}}$ starts at the $4 \mathrm{PN}$ level. The $4 \mathrm{PN}$-accurate value of $S_{\text {nonloc }}$ was obtained in Ref. [2] (see also the related works 20 22]). The 5PNaccurate value of $S_{\text {nonloc }}$ was obtained in section IXA of [23] (based on Ref. [24]). [See also Refs. [25, 26] for the related 5PN logarithmic terms.] It reads, from Eq. (9.12) of [23] (see also the related recent work [27]),

$$
\begin{aligned}
S_{\text {nonloc }}^{4+5 \mathrm{PN}}\left[x_{1}\left(s_{1}\right), x_{2}\left(s_{2}\right)\right]= & \frac{G^{2} \mathcal{M}}{c^{3}} \int d t \mathrm{Pf}_{2 r_{12}^{h}(t) / c} \times \\
& \int \frac{d t^{\prime}}{\left|t-t^{\prime}\right|} \mathcal{F}_{1 \mathrm{PN}}^{\mathrm{split}}\left(t, t^{\prime}\right) .
\end{aligned}
$$

Here, $\mathcal{M}$ denotes the total conserved mass-energy of the binary system, while $\mathcal{F}_{1 \mathrm{PN}}^{\text {split }}\left(t, t^{\prime}\right)$ denotes the time-split version of the fractionally $1 \mathrm{PN}$-accurate gravitationalwave energy flux emitted by the system, namely (using a superscript in parenthesis to denote a repeated timederivative)

$$
\begin{aligned}
& \mathcal{F}_{1 \mathrm{PN}}^{\mathrm{split}}\left(t, t^{\prime}\right)=\frac{G}{c^{5}}\left(\frac{1}{5} I_{a b}^{(3)}(t) I_{a b}^{(3)}\left(t^{\prime}\right)\right. \\
& \left.+\frac{1}{189 c^{2}} I_{a b c}^{(4)}(t) I_{a b c}^{(4)}\left(t^{\prime}\right)+\frac{16}{45 c^{2}} J_{a b}^{(3)}(t) J_{a b}^{(3)}\left(t^{\prime}\right)\right)
\end{aligned}
$$

The mass and spin multipole moments $I_{a b}, I_{a b c}, J_{a b}$, entering the latter expression are the Blanchet-Damour (1PN-accurate) source multipole moments defined by explicit integrals over the stress-energy tensor of the source [17]. Their explicit expressions for a binary system can be found in Ref. [28]. Eq. (55) defines an explicit functional of the two worldlines, and subtracting it from the (in principle PM-computable) total action $S_{\text {tot }}$ defines the local-in-time contribution $S_{\mathrm{loc}}^{\leq 5 \mathrm{PN}}$ to the twobody dynamics. The time-scale entering the partie finie operation (Pf) used in (5) to define the logarithmically divergent integral over $t^{\prime}$ has been fixed to be $2 r_{12}^{h} / c$, where $r_{12}^{h}$ denotes the (harmonic-coordinate) radial distance between the two bodies. Note that the meaning here of $S_{\text {loc }}$ (and its corresponding $H_{\text {loc }}$ ) differs from the one in Refs. [13, 23], where $H_{\text {loc }}$ included logarithmic contributions in its definition.

Second step: computing the $O(\nu)$ piece of the timeaveraged redshift $\left\langle z_{1}\right\rangle$ to sixth order in eccentricity - The second step of our approach consists in using gravitational Self-Force (SF) theory to compute to sufficient accuracy the first-order-self-force (1SF) contribution, say $\delta z_{1}=O(\nu)$, to the time-averaged redshift $\left\langle z_{1}\right\rangle=\left\langle d s_{1} / d t\right\rangle$ of the first body, considered as a function of the symmetric mass ratio $\nu$ and of the dimensionless radial and azimuthal frequencies $M \Omega_{r}, M \Omega_{\phi}$ of eccentric orbits [29, 30]. [We denote $M \equiv m_{1}+m_{2}, \mu=m_{1} m_{2} / M$, $\nu=\mu / M=m_{1} m_{2} /\left(m_{1}+m_{2}\right)^{2}$.] Ref. 31] has developed efficient tools for analytically computing $\delta z_{1}$ as a function of the inverse parameter of the elliptical orbit, $u_{p}=G M /\left(c^{2} p\right)$, and of the eccentricity, $e$. Current results reached either high-orders in $e$ limited to $4 \mathrm{PN}$ accuracy 32], or high PN accuracy limited to fourth order in $e$ [33]. We crucially needed, for the present work, to extend the computation of $\delta z_{1}$ to the sixth order in $e$, and, to, at least, the $5 \mathrm{PN}$ accuracy, i.e., the sixth order in $u_{p}$. The result of our computation for the coefficient of $e^{6}$ in $\delta z_{1}$ reads, at $5 \mathrm{PN}$ accuracy,

$$
\begin{aligned}
\delta z_{1}^{e^{6}}= & \nu\left[\frac{1}{4} u_{p}^{3}+\left(-\frac{53}{12}-\frac{41}{128} \pi^{2}\right) u_{p}^{4}\right. \\
& \left.+C_{5} u_{p}^{5}+C_{6} u_{p}^{6}+o\left(u_{p}^{6}\right)\right]+O\left(\nu^{2}\right),
\end{aligned}
$$

where

$$
\begin{aligned}
C_{5}= & -\frac{38471}{360}+\frac{6455}{4096} \pi^{2}-\frac{178288}{5} \ln (2) \\
& +\frac{1994301}{160} \ln (3)+\frac{1953125}{288} \ln (5)+16 \gamma+8 \ln \left(u_{p}\right), \\
C_{6}= & -\frac{17344111}{5040}+\frac{782899}{4096} \pi^{2}+\frac{66668054}{135} \ln (2) \\
& -\frac{29268135}{448} \ln (3)-\frac{2027890625}{12096} \ln (5) \\
& -\frac{1694}{5} \gamma-\frac{847}{5} \ln \left(u_{p}\right) .
\end{aligned}
$$

We have also determined the higher-order contributions in $u_{p}$ up to $u_{p}^{19 / 2}$ 34. 
Third step: using the first law of binary dynamics to translate $\delta z_{1}^{e^{6}}$ into a corresponding $O\left(p_{r}^{6}\right)$-accurate, effective-one-body Hamiltonian - The first law of binary dynamics [26, 35, 36] allows one to transform the gaugeinvariant information contained in our new result (7) (together with the previous $O\left(e^{4}\right)$ results [32, 33]) into a corresponding knowledge of the (gauge-fixed) two-body Hamiltonian, as expressed in effective-one-body (EOB) theory [37, 38]. To do this we had to extend the results of [36] to the sixth order in the ( $\mu$-rescaled) radial momentum $p_{r}$. EOB theory expresses the two-body Hamiltonian $H\left(=\mathcal{M} c^{2}\right)$ in terms of a rescaled "effective" Hamiltonian $\widehat{H}_{\text {eff }}$ according to

$$
H=M c^{2} \sqrt{1+2 \nu\left(\widehat{H}_{\mathrm{eff}}-1\right)} .
$$

In turn, $\widehat{H}_{\text {eff }}$ is expressed in terms of various bricks: two radial potentials $A(u ; \nu)$, and $\bar{D}(u ; \nu) \equiv$ $(A(u ; \nu) B(u ; \nu))^{-1}$, and a momentum-dependent potential $Q(u, p ; \nu)$, where $u \equiv G M /\left(c^{2} r\right)$. Namely, henceforth setting $c=1$,

$\widehat{H}_{\mathrm{eff}}^{2}=A(u ; \nu)\left[1+A(u ; \nu) \bar{D}(u ; \nu) p_{r}^{2}+p_{\phi}^{2} u^{2}+Q(u, p ; \nu)\right]$.

The PN expansions of the potentials $A(u ; \nu)$, and $\bar{D}(u ; \nu)$ are written as $A(u ; \nu)=1-2 u+\sum_{n} a_{n}(\nu, \ln u) u^{n}$ and $\bar{D}(u ; \nu)=1+\sum_{n} \bar{d}_{n}(\nu, \ln u) u^{n}$. In the gauge (hereafter called " $p_{r}$ gauge") introduced in [38], the PN expansion of $Q(u, p)$ is given by a double expansion in $u$ and $p_{r}^{2}$, say $Q=q_{4}(u ; \nu) p_{r}^{4}+q_{6}(u ; \nu) p_{r}^{6}+q_{8}(u ; \nu) p_{r}^{8}+\cdots$, where $q_{m}(u ; \nu)=\sum_{n} q_{m n}(\nu, \ln u) u^{n}$. In addition, all the (logarithmically running) $\nu$-dependent coefficients $a_{n}(\nu, \ln u)$, $\bar{d}_{n}(\nu, \ln u), q_{m n}(\nu, \ln u)$ are polynomials in $\nu$, starting at $\nu^{1}$, and of degree increasing with $n$. We derived the relation linking the $1 \mathrm{SF}(O(\nu))$ piece in $q_{6}(u ; \nu)$ to the $1 \mathrm{SF}$ redshift $\delta z_{1}\left(u_{p}, e\right)=\delta z_{1}^{e^{0}}\left(u_{p}\right)+\delta z_{1}^{e^{2}}\left(u_{p}\right) e^{2}+\delta z_{1}^{e^{4}}\left(u_{p}\right) e^{4}+$ $\delta z_{1}^{e^{6}}\left(u_{p}\right) e^{6}$. This allowed us to extend the previous $1 \mathrm{SF}$ knowledge of $q_{4}(u ; \nu) p_{r}^{4}$ 33] to the $p_{r}^{6}$ level, namely

$$
q_{6}(u ; \nu)=\nu q_{62}^{\nu^{1}} u^{2}+\nu q_{63}^{\nu^{1}} u^{3}+O\left(u^{7 / 2}\right)+O\left(\nu^{2}\right),
$$

where $q_{62}^{\nu^{1}}$ is a known $4 \mathrm{PN}$ term [23] and where

$$
\begin{aligned}
q_{63}^{\nu^{1}} & =\frac{2613083}{1050}+\frac{6875745536}{4725} \ln (2) \\
& -\frac{23132628}{175} \ln (3)-\frac{101687500}{189} \ln (5),
\end{aligned}
$$

is a new, $5 \mathrm{PN}$ level, result. See Ref. 34] for the higherorder contributions in $u$ (up to $u^{19 / 2}$ included).

Fourth step: determining the $1 S F$ contribution to the local-in-time $5 \mathrm{PN}$-accurate Hamiltonian by subtracting the nonlocal action - Inserting our new result (11), together with the previous high-PN 1SF knowledge of $A(u ; \nu), \bar{D}(u ; \nu)$ and $q_{4}(u ; \nu)$, in Eqs. (9) and (10) determines the two-body Hamiltonian at the combined 1SF $+5 \mathrm{PN}$ accuracy. [At the level of the unrescaled, total Hamiltonian $H$, Eq. (9), 1SF accuracy means knowing both the $\nu^{1}$ and the $\nu^{2}$ contributions.] We can then subract from the full Hamiltonian action $\int p d q-H(q, p) d t$ the nonlocal-in-time term (5) to compute the local-intime Hamiltonian action $\int p d q-H_{\mathrm{loc}}(q, p) d t$. This is conveniently done by using the Delaunay averaging technique of the nonlocal action introduced in [23]. This averaging technique leads to a gauge-invariant result which can then be expressed in the EOB- $p_{r}$ gauge. The so obtained $1 \mathrm{SF}+5 \mathrm{PN}$ accurate local-in-time Hamiltonian $H_{\text {loc }}(q, p)$ can then be expressed (via the universal EOB energy map (9) ) in terms of corresponding 1SF + $5 \mathrm{PN}$ accurate EOB potentials $A_{\mathrm{loc}}(u ; \nu), \bar{D}_{\mathrm{loc}}(u ; \nu)$ and $Q_{\text {loc }}\left(u, p_{r} ; \nu\right)$. All logarithmic dependence (including numerical logs, like $\ln 2$ ) has disappeared from these local potentials. For instance, the local contribution to $q_{6}(u ; \nu)$ was found to be $q_{6}^{\text {loc }}=-\frac{9}{5} \nu u^{2}+\frac{123}{10} \nu u^{3}+O\left(\nu^{2}\right)$. Here, the contribution $+\frac{123}{10} \nu u^{3}$ is at the $5 \mathrm{PN}$ level.

Fifth step: using EOB-PM theory to determine most of the nonlinear dependence on $\nu$ of the local HamiltonianAt this stage, while we know the exact dependence of the nonlocal action (5) on the two masses $m_{1}, m_{2}$, and therefore on $\nu$ for a given $M$, our use of SF technology has limited our determination of the local Hamiltonian $H_{\mathrm{loc}}(q, p ; \nu)$ to the $1 \mathrm{SF}$ accuracy: $H_{\mathrm{loc}}=M c^{2}+\nu H_{\mathrm{loc}}^{(1)}+$ $\nu^{2} H_{\mathrm{loc}}^{(2)}+O\left(\nu^{3}\right)$. We can, however, determine most of the higher-order powers in $\nu$ by using results from the EOB formalism applied to PM-expanded scattering. More precisely, we can use two constraints.

On the one hand, the exact $\nu$ dependence of the EOB Hamiltonian has been determined both at the first postMinkowskian (1PM) level 39], and at the second postMinkowskian (2PM) level [40]. By transforming the latter results (obtained in a special "energy" gauge) into the (standard) EOB- $p_{r}$ gauge used above, we can determine the exact $\nu$ dependence of the $5 \mathrm{PN}$-accurate (local and nonlocal) Hamiltonian for all the terms in the Hamiltonian which are either $\propto u^{1}$ or $\propto u^{2}$. For instance, we thereby found that the coefficient $q_{82}$ of $p_{r}^{8} u^{2}$ in the $Q$ potential is $q_{82}=q_{82}^{\mathrm{loc}}=\frac{6}{7} \nu+\frac{18}{7} \nu^{2}+\frac{24}{7} \nu^{3}-6 \nu^{4}$.

On the other hand, the general dictionary [40] between the EOB Hamiltonian and the PM-expanded scattering function, $\frac{1}{2} \chi\left(\widehat{\mathcal{E}}_{\text {eff }}, j\right)=\sum_{n} \chi_{n}\left(\widehat{\mathcal{E}}_{\text {eff }}\right) / j^{n}$, where $j \equiv J /\left(G m_{1} m_{2}\right)$, has recently been used [41] to show that the combination $\left(1+2 \nu\left(\widehat{\mathcal{E}}_{\text {eff }}-1\right)\right)^{n-1} \chi_{n}\left(\widehat{\mathcal{E}}_{\text {eff }}\right)$ was a polynomial in $\nu$ of degree $d(n)$ equal to the integer part of $(n-1) / 2$. This yields a strong restriction on the $\nu$ dependence of the coefficients of the 5PN-level local Hamiltonian,

$$
H_{\mathrm{loc}}^{5 \mathrm{PN}}=\sum_{m+n=6} h_{2 m n}(\nu)\left(p^{2}\right)^{m} u^{n} .
$$

[For notational simplicity, we use in Eq. (13) $p^{2}$ to denote either $p_{r}^{2}$ or $p_{t}^{2} \equiv p_{\phi}^{2} / r^{2}$.] In order to apply this restriction, we computed (as a function of the coefficients $\left.h_{2 m n}(\nu)\right)$ the scattering angle implied by the total, 5PNaccurate Hamiltonian (using the technique of Ref. [13]). 


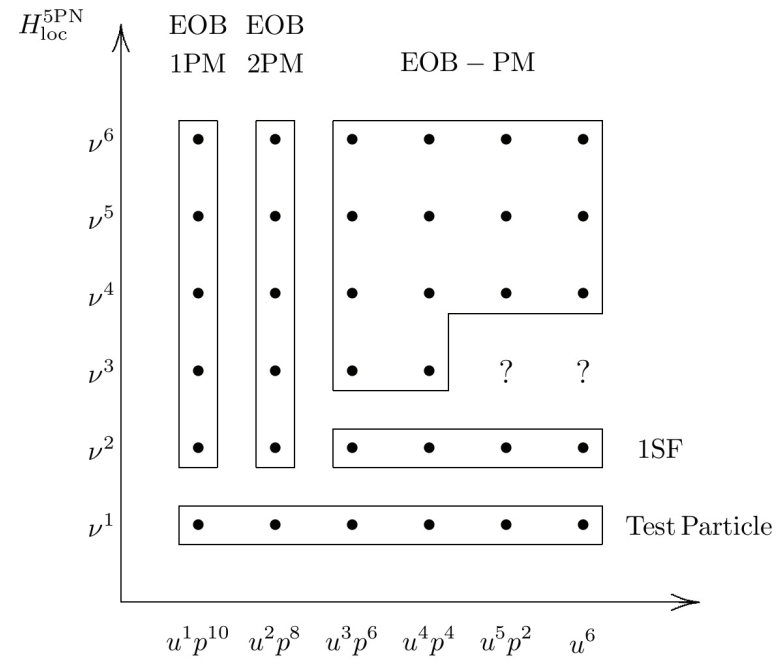

FIG. 1: Schematic representation of the theoretical tools used to obtain the various contributions to the $5 \mathrm{PN}$-accurate local Hamiltonian. These contributions are keyed, on the horizontal axis, by powers of $u=G M / r$ and squared momentum $p^{2} \sim p_{r}^{2} \sim p_{t}^{2}$, and, on the the vertical axis, by powers of $\nu \equiv m_{1} m_{2} /\left(m_{1}+m_{2}\right)^{2}$. The bullets indicate the coefficients determined for the first time in the present work. The question marks denote the only two missing coefficients.

Final result for the local-in-time $5 P N$-accurate Hamiltonian - The 5PN-level local Hamiltonian, Eq. (13), a priori contains (in our gauge) 36 unknown numerical coefficients, say $h_{2 m n}^{\nu^{k}}$ parametrizing the powers of $\nu$ in the various coefficients $h_{2 m n}(\nu)=\sum_{k=1}^{k_{\max }(m, n)} h_{2 m n}^{\nu^{k}} \nu^{k}$ appearing in Eq. (13). [Here, we do not distinguish the coefficients of $p_{r}^{2}$ or $p_{t}^{2}$. If distinguished, there are 108 coefficients.] The degrees of these polynomials in $\nu$ are indeed found (when $m+n=6$ ) to be all equal to $k_{\text {max }}=6$ when $n=6-m=1, \ldots, 6$.

Combining all the previous tools and results, we were able to determine 34 of the a priori unknown numerical coefficients $h_{2 m n}^{\nu^{k}}$. Fig. 1 indicates the source of information having allowed us to determine each one of these 34 coefficients: the test-particle limit determines the $\nu^{1}$ row; the $1 \mathrm{SF}$ computations determine the $\nu^{2}$ row; the first two columns are respectively determined by the $1 \mathrm{PM}$ and 2PM exact EOB Hamiltonians; the $\nu^{\geq 3}$ dependence of the next third and fourth columns (respectively corresponding to $3 \mathrm{PM}$ and $4 \mathrm{PM}$ ) are completely determined by the EOB-PM scattering constraint mentioned above. The latter constraint determines the coefficients in the last two columns (5PM and 6PM) except for the two coefficients $h_{25}^{\nu^{3}}$ and $h_{06}^{\nu^{3}}$. [Distinguishing $p_{r}^{2}$ and $p_{t}^{2}$, we determine 106 coefficients among 108.] When using the (more compact) EOB parametrization of the local Hamiltonian the full description of the local-in-time $5 \mathrm{PN}$-accurate Hamiltonian $H_{\mathrm{loc}}^{5 \mathrm{PN}}$ is obtained by inserting in the EOB map (9) the effective Hamiltonian $\widehat{H}_{\text {eff }}$ de- fined by the following (logarithm-free) values of the local pieces of the EOB building blocks $A(u ; \nu), \bar{D}(u ; \nu)$ and $Q\left(u, p_{r} ; \nu\right)$ :

$$
\begin{aligned}
A_{\mathrm{loc}}= & 1-2 u+2 \nu u^{3}+\nu\left(\frac{94}{3}-\frac{41}{32} \pi^{2}\right) u^{4} \\
& +a_{5}^{\mathrm{loc}} u^{5}+a_{6}^{\mathrm{loc}} u^{6}, \\
\bar{D}_{\mathrm{loc}}= & 1+6 \nu u^{2}+\left(52 \nu-6 \nu^{2}\right) u^{3}+\bar{d}_{4}^{\mathrm{loc}} u^{4}+\bar{d}_{5}^{\mathrm{loc}} u^{5}, \\
Q_{\mathrm{loc}}= & p_{r}^{4}\left[2(4-3 \nu) \nu u^{2}+q_{43}^{\mathrm{loc}} u^{3}+q_{44}^{\mathrm{loc}} u^{4}\right] \\
& +p_{r}^{6}\left(q_{62}^{\mathrm{loc}} u^{2}+q_{63}^{\mathrm{loc}} u^{3}\right)+q_{82}^{\mathrm{loc}} p_{r}^{8} u^{2},
\end{aligned}
$$

with

$$
\begin{aligned}
a_{5}^{\text {loc }}= & \left(-\frac{4237}{60}+\frac{2275}{512} \pi^{2}\right) \nu+\left(\frac{41}{32} \pi^{2}-\frac{221}{6}\right) \nu^{2} \\
a_{6}^{\text {loc }}= & \left(-\frac{1026301}{1575}+\frac{246367}{3072} \pi^{2}\right) \nu+a_{6}^{\nu^{2}} \nu^{2} \\
& +4 \nu^{3} \\
\bar{d}_{4}^{\text {loc }}= & \left(\frac{1679}{9}-\frac{23761}{1536} \pi^{2}\right) \nu+\left(-260+\frac{123}{16} \pi^{2}\right) \nu^{2} \\
\bar{d}_{5}^{\text {loc }}= & \left(\frac{331054}{175}-\frac{63707}{512} \pi^{2}\right) \nu+\bar{d}_{5}^{\nu^{2}} \nu^{2} \\
+ & \left(\frac{1069}{3}-\frac{205}{16} \pi^{2}\right) \nu^{3}
\end{aligned}
$$

and

$$
\begin{aligned}
q_{43}^{\mathrm{loc}} & =20 \nu-83 \nu^{2}+10 \nu^{3} \\
q_{44}^{\mathrm{loc}} & =\left(\frac{1580641}{3150}-\frac{93031}{1536} \pi^{2}\right) \nu \\
& +\left(-\frac{2075}{3}+\frac{31633}{512} \pi^{2}\right) \nu^{2}+\left(640-\frac{615}{32} \pi^{2}\right) \nu^{3} \\
q_{62}^{\mathrm{loc}} & =-\frac{9}{5} \nu-\frac{27}{5} \nu^{2}+6 \nu^{3} \\
q_{63}^{\mathrm{loc}} & =\frac{123}{10} \nu-\frac{69}{5} \nu^{2}+116 \nu^{3}-14 \nu^{4} \\
q_{82}^{\mathrm{loc}} & =\frac{6}{7} \nu+\frac{18}{7} \nu^{2}+\frac{24}{7} \nu^{3}-6 \nu^{4} .
\end{aligned}
$$

Modulo the two undetermined coefficients $a_{6}^{\nu^{2}}$ and $\bar{d}_{5}^{\nu^{2}}$, the full $5 \mathrm{PN}$-accurate dynamics is given by adding to the local action defined by $H_{\mathrm{loc}}^{\leq 5 \mathrm{PN}}$ the $4+5 \mathrm{PN}$ nonlocal one Eq. (5).

New results at $3 P M$ and $4 P M-$ As one can see on Fig. 1, our results give a complete description of the $5 \mathrm{PN}$ dynamics at the 3PM and 4PM levels (fourth and fifth columns in Fig. 1). This means in particular that our findings allow us to compute, with $5 \mathrm{PN}$ accuracy, the $3 \mathrm{PM}\left(O\left(G^{3}\right)\right)$ and $4 \mathrm{PM}\left(O\left(G^{4}\right)\right)$ terms, $\chi_{3}$ and $\chi_{4}$, in the scattering angle. The computation at $5 \mathrm{PN}$ accuracy of $\chi_{3}$ from our results for the full loc + nonloc dynamics $\left(\right.$ with $\left.\chi_{3}^{\text {nonloc }}=0[13]\right)$ yields $\left(\right.$ denoting $\left.p_{\infty} \equiv \sqrt{\widehat{\mathcal{E}}_{\text {eff }}^{2}-1}\right)$

$$
\chi_{3}=-\frac{1}{3 p_{\infty}^{3}}+\frac{4}{p_{\infty}}+(-8 \nu+24) p_{\infty}
$$




$$
\begin{aligned}
& +\left(-36 \nu+\frac{64}{3}+8 \nu^{2}\right) p_{\infty}^{3} \\
& +\left(-\frac{91}{5} \nu+34 \nu^{2}-8 \nu^{3}\right) p_{\infty}^{5} \\
& +\left(\frac{69}{70} \nu+\frac{51}{5} \nu^{2}-32 \nu^{3}+8 \nu^{4}\right) p_{\infty}^{7}+o\left(p_{\infty}^{7}\right) \cdot(1
\end{aligned}
$$

In this expression the last term $\propto p_{\infty}^{7}$ is the $5 \mathrm{PN}$ contribution to $\chi_{3}$. Importantly, we checked that this newly derived result is in agreement with the corresponding $5 \mathrm{PN}-$ level term in the PN expansion of the (partly conjectural) $3 \mathrm{PM}$-level recent result of [11, 12]. This is the first independent, partial confirmation of the latter result.

In addition, our results yield an explicit 5PN-accurate value for the 4 PM-level scattering angle $\chi_{4}=\chi_{4}^{\text {loc }}+$ $\chi_{4}^{\text {nonloc }}$. Let us only cite here the 5PN-level term in the local contribution $\chi_{4}^{\text {loc }}\left(p_{\infty}\right)$ :

$$
\begin{gathered}
\chi_{4 \text { loc }}^{5 \mathrm{PN}}\left(p_{\infty}\right)=\pi\left(-\frac{94899}{32768} \pi^{2} \nu^{2}+\frac{93031}{32768} \pi^{2} \nu\right. \\
-\frac{1945583}{33600} \nu+\frac{1937}{16} \nu^{2}-\frac{2895}{32} \nu^{3}+\frac{525}{64} \nu^{4} \\
\left.+\frac{1845}{2048} \pi^{2} \nu^{3}\right) p_{\infty}^{6} .
\end{gathered}
$$

The complementary nonlocal contribution is derivable by the methods of [13].

Conclusions. - We have introduced a new methodology (based on combining several different theoretical tools) for analytically computing the conservative dynamics of two bodies in General Relativity. We have applied our approach to deriving a nearly complete expression for the $5 \mathrm{PN}$-level action. It is given by the sum of a $4 \mathrm{PN}+5 \mathrm{PN}$ nonlocal action, Eq. (5), and of a local one $\int p d q-H_{\mathrm{loc}}^{\leq 5 \mathrm{PN}} d t$. We determined the full functional structure of $H_{\mathrm{loc}}^{\leq 5 \mathrm{PN}}$, except for two $\left(\nu^{3}\right.$-level) unknown coefficients. Our results give access to the $5 \mathrm{PN}$-accurate $O\left(G^{3}\right)$ and $O\left(G^{4}\right)$ scattering angles. This provided the first independent confirmation of the recent 3PM result of Refs. [11, 12].

Our work opens promising avenues for further progress on the dynamics of binary systems. Indeed, the technique we defined here can be extended, in principle, to higher PN orders. Our work also offers new motivations for doing targeted, partial computations able to determine the two currently missing numerical coefficients. We can think of several ways in which they could be determined: second-order self-force computation; partial computation of 5PN dynamics by traditional techniques aiming only at terms having selected mass dependence; or, eventually, high-accuracy numerical computation.
[1] B. P. Abbott et al. [LIGO Scientific and Virgo Collaborations], "GWTC-1: A Gravitational-Wave Transient Catalog of Compact Binary Mergers Observed by LIGO and Virgo during the First and Second Observing Runs," arXiv:1811.12907 [astro-ph.HE].

[2] T. Damour, P. Jaranowski and G. Schäfer, "Nonlocal-intime action for the fourth post-Newtonian conservative dynamics of two-body systems," Phys. Rev. D 89, no. 6, 064058 (2014) arXiv:1401.4548 [gr-qc]].

[3] P. Jaranowski and G. Schäfer, "Derivation of local-intime fourth post-Newtonian ADM Hamiltonian for spinless compact binaries," Phys. Rev. D 92, no. 12, 124043 (2015) arXiv:1508.01016 [gr-qc]].

[4] L. Bernard, L. Blanchet, A. Bohé, G. Faye and S. Marsat, "Fokker action of nonspinning compact binaries at the fourth post-Newtonian approximation," Phys. Rev. D 93, no. 8, 084037 (2016) arXiv:1512.02876] [gr-qc]].

[5] T. Damour, P. Jaranowski and G. Schäfer, "Conservative dynamics of two-body systems at the fourth postNewtonian approximation of general relativity," Phys. Rev. D 93, no. 8, 084014 (2016) arXiv:1601.01283 [gr$\mathrm{qc}]]$.

[6] T. Marchand, L. Bernard, L. Blanchet and G. Faye, "Ambiguity-Free Completion of the Equations of Motion of Compact Binary Systems at the Fourth PostNewtonian Order," Phys. Rev. D 97, no. 4, 044023 (2018) arXiv:1707.09289 [gr-qc]].

[7] S. Foffa and R. Sturani, "Conservative dynamics of binary systems to fourth Post-Newtonian order in the EFT approach I: Regularized Lagrangian," Phys. Rev. D 100, no. 2, 024047 (2019) arXiv:1903.05113 [gr-qc]].

[8] S. Foffa, R. A. Porto, I. Rothstein and R. Sturani, "Conservative dynamics of binary systems to fourth PostNewtonian order in the EFT approach II: Renormalized Lagrangian," Phys. Rev. D 100, no. 2, 024048 (2019) arXiv:1903.05118 [gr-qc]].

[9] S. Foffa, P. Mastrolia, R. Sturani, C. Sturm and W. J. Torres Bobadilla, "Static two-body potential at fifth post-Newtonian order," Phys. Rev. Lett. 122, no. 24, 241605 (2019) arXiv:1902.10571 [gr-qc]].

[10] J. Blümlein, A. Maier and P. Marquard, "Five-Loop Static Contribution to the Gravitational Interaction Potential of Two Point Masses," arXiv:1902.11180 [gr-qc].

[11] Z. Bern, C. Cheung, R. Roiban, C. H. Shen, M. P. Solon and M. Zeng, "Scattering Amplitudes and the Conservative Hamiltonian for Binary Systems at Third PostMinkowskian Order," Phys. Rev. Lett. 122, no. 20, 201603 (2019) arXiv:1901.04424 [hep-th]].

[12] Z. Bern, C. Cheung, R. Roiban, C. H. Shen, M. P. Solon and M. Zeng, "Black Hole Binary Dynamics from the Double Copy and Effective Theory," arXiv:1908.01493 [hep-th].

[13] D. Bini and T. Damour, "Gravitational scattering of two black holes at the fourth post-Newtonian approximation," Phys. Rev. D 96, no. 6, 064021 (2017) arXiv:1706.06877 [gr-qc]].

[14] T. Damour and G. Esposito-Farese, "Testing gravity to second postNewtonian order: A Field theory approach," Phys. Rev. D 53, 5541 (1996) gr-qc/9506063.

[15] G. Schäfer and P. Jaranowski, "Hamiltonian formula- 
tion of general relativity and post-Newtonian dynamics of compact binaries," Living Rev. Rel. 21, no. 1, 7 (2018) arXiv:1805.07240 [gr-qc]].

[16] L. Blanchet and T. Damour, "Tail Transported Temporal Correlations in the Dynamics of a Gravitating System," Phys. Rev. D 37, 1410 (1988).

[17] L. Blanchet and T. Damour, "Postnewtonian Generation of Gravitational Waves," Ann. Inst. H. Poincare Phys. Theor. 50, 377 (1989).

[18] O. Poujade and L. Blanchet, "PostNewtonian approximation for isolated systems calculated by matched asymptotic expansions," Phys. Rev. D 65, 124020 (2002) gr-qc/0112057.

[19] L. Blanchet and T. Damour, "Radiative gravitational fields in general relativity I. general structure of the field outside the source," Phil. Trans. Roy. Soc. Lond. A 320, 379 (1986).

[20] S. Foffa and R. Sturani, "Tail terms in gravitational radiation reaction via effective field theory," Phys. Rev. D 87, no. 4, 044056 (2013) arXiv:1111.5488 [gr-qc]].

[21] A. Ross, "Multipole expansion at the level of the action," Phys. Rev. D 85, 125033 (2012) arXiv:1202.4750 [gr-qc]].

[22] C. R. Galley, A. K. Leibovich, R. A. Porto and A. Ross, "Tail effect in gravitational radiation reaction: Time nonlocality and renormalization group evolution," Phys. Rev. D 93, 124010 (2016) arXiv:1511.07379 [gr-qc]].

[23] T. Damour, P. Jaranowski, and G. Schäfer, "Fourth postNewtonian effective one-body dynamics," Phys. Rev. D 91, 084024 (2015) arXiv:1502.07245 [gr-qc]].

[24] T. Damour (unpublished); cited in L. Barack, T. Damour, and N. Sago, "Precession effect of the gravitational self-force in a Schwarzschild spacetime and the effective one-body formalism", Phys. Rev. D 82, 084036 (2010), which quoted and used some combinations of the (4PN and 5PN) logarithmic contributions to the EOB potentials $A(u)$ and $\bar{D}(u)$.

[25] L. Blanchet, S. L. Detweiler, A. Le Tiec and B. F. Whiting, "High-Order Post-Newtonian Fit of the Gravitational Self-Force for Circular Orbits in the Schwarzschild Geometry," Phys. Rev. D 81, 084033 (2010) arXiv:1002.0726 [gr-qc]].

[26] A. Le Tiec, L. Blanchet and B. F. Whiting, "The First Law of Binary Black Hole Mechanics in General Relativity and Post-Newtonian Theory," Phys. Rev. D 85, 064039 (2012) arXiv:1111.5378 [gr-qc]].

[27] S. Foffa and R. Sturani, "Hereditary Terms at Next-ToLeading Order in Two-Body Gravitational Dynamics," arXiv:1907.02869 [gr-qc].

[28] L. Blanchet and G. Schäfer, "Higher order gravitational radiation losses in binary systems," Mon. Not. Roy. Astron. Soc. 239, 845 (1989) Erratum: [Mon. Not. Roy. Astron. Soc. 242, 704 (1990)].

[29] S. L. Detweiler, "A Consequence of the gravitational selfforce for circular orbits of the Schwarzschild geometry," Phys. Rev. D 77, 124026 (2008) arXiv:0804.3529 [gr-qc]].

[30] L. Barack and N. Sago, "Beyond the geodesic approximation: conservative effects of the gravitational self-force in eccentric orbits around a Schwarzschild black hole," Phys. Rev. D 83, 084023 (2011) arXiv:1101.3331 [grqc]].

[31] D. Bini and T. Damour, "Analytical determination of the two-body gravitational interaction potential at the fourth post-Newtonian approximation," Phys. Rev. D 87, no. 12, 121501 (2013) arXiv:1305.4884 [gr-qc]].

[32] S. Hopper, C. Kavanagh and A. C. Ottewill, "Analytic self-force calculations in the post-Newtonian regime: eccentric orbits on a Schwarzschild background," Phys. Rev. D 93, no. 4, 044010 (2016) arXiv:1512.01556 [grqc]].

[33] D. Bini, T. Damour and A. Geralico, "New gravitational self-force analytical results for eccentric orbits around a Schwarzschild black hole," Phys. Rev. D 93, no. 10, 104017 (2016) arXiv:1601.02988 [gr-qc]].

[34] D. Bini, T. Damour and A. Geralico, in preparation.

[35] E. Barausse, A. Buonanno and A. Le Tiec, "The complete non-spinning effective-one-body metric at linear order in the mass ratio," Phys. Rev. D 85, 064010 (2012) arXiv:1111.5610 [gr-qc]].

[36] A. Le Tiec, "First Law of Mechanics for Compact Binaries on Eccentric Orbits," Phys. Rev. D 92, no. 8, 084021 (2015) arXiv:1506.05648 [gr-qc]].

[37] A. Buonanno and T. Damour, "Effective one-body approach to general relativistic two-body dynamics," Phys. Rev. D 59, 084006 (1999) arXiv:gr-qc/9811091.

[38] T. Damour, P. Jaranowski, and G. Schäfer, "On the determination of the last stable orbit for circular general relativistic binaries at the third post-Newtonian approximation," Phys. Rev. D 62, 084011 (2000) arXiv:gr-qc/0005034.

[39] T. Damour, "Gravitational scattering, post-Minkowskian approximation and Effective One-Body theory," Phys. Rev. D 94, no. 10, 104015 (2016) arXiv:1609.00354] [grqc]].

[40] T. Damour, "High-energy gravitational scattering and the general relativistic two-body problem," Phys. Rev. D 97, no. 4, 044038 (2018) arXiv:1710.10599 [gr-qc]].

[41] T. Damour, in preparation. 